\title{
SCIENTIFIC EVALUATION OF POLYCLINIC OPERATING STRATEGIES WITH DISCRETE-EVENT SIMULATION
}

\author{
Oh, H. C. \& Chow, W. L. \\ Centre for Health Services Research, Singapore Health Services Pte Ltd. \\ 168 Jalan Bukit Merah \#06-08 Tower 3, Singapore 150168 \\ E-Mail: oh.hong.choon@singhealth.com.sg
}

\begin{abstract}
A subsidized primary care clinic in Singapore wanted to evaluate the impact of different patient appointment apportionment and patient-doctor allocation strategies on the cycle time that their patients need to spend during their clinic visits. To the best of the authors' knowledge, there is limited literature on such studies. This paper aims to fill this research gap via scientific evaluation of these operating strategies. Based on simulation model projections, two key inferences are made. First, appointment system is a good patient classification strategy that reduces median and $95^{\text {th }}$ percentile cycle times of appointment patients. But the magnitude of these reductions in median and $95^{\text {th }}$ percentile cycle times diminish as the proportion of appointment patients in a patient population increases. Second, exclusive allocation of walk-in patients seeking consultation for their non-chronic conditions to selected doctors is not effective relative to appointment systems in reducing overall median and $95^{\text {th }}$ percentile cycle times.

(Received in September 2010, accepted in September 2011. This paper was with the authors 1 month for 2 revisions.)
\end{abstract}

Key Words: Cycle Time, Discrete-Event Simulation, Appointment Scheduling, Walk-in Patients

\section{INTRODUCTION}

Primary health care entails the provision of primary medical treatment, preventive healthcare and health education to the local community. In Singapore, about $20 \%$ of primary health care is provided through an island network of 18 outpatient primary care centres (polyclinics) that provide heavily subsidized primary care to those seeking treatment. The remaining $80 \%$ is provided through some 2000 private medical practitioner's clinics which can be located within business or shopping districts or among residential neighbourhoods. Each polyclinic serves as a one-stop health centre that provides government-subsidized outpatient medical care, followup of patients discharged from hospitals, immunization, health screening and education, investigative facilities and pharmacy services. In general, the costs incurred at polyclinics are low as compared to visits to private medical practitioners.

As a result of the highly affordable healthcare charges, polyclinics attract large patient attendance volumes which result in relatively long wait to see doctors by the patients during their polyclinic visits $[1,2]$. In an effort to improve patient satisfaction, the polyclinics have implemented several measures over the years to enhance their health care service delivery. These measures include installation of self-service queue ticketing machines; Short Message Service (SMS) notification system, which sends an SMS to the patient shortly before his or her queue number is called; online web-cam queue watch which allows patients to check the real time queue lengths at various counters of all polyclinics. 
To this end, it is important to note that the workflow processes of all polyclinics in Singapore are not uniform as the heads of the polyclinics are given the autonomy to run their clinics in manners which they deem fit for their patients. For example, there are polyclinics which only receive patients with appointments (or pre-scheduled times) while majority of others still have walk-in patients (who could generally visit the clinics at any time within their operating hours) contributing to $100 \%$ of their patient attendances.

In their continual effort to improve their health care service delivery, the management of a local polyclinic wanted to evaluate the operational feasibility of patient appointment system and new patient-doctor allocation strategies. Currently, the polyclinic concerned allocated approximately $10 \%$ of its patients to see senior doctors or nurses on a partial appointment system for their chronic diseases while the remaining patient population are walk-in patients. In particular, the polyclinic management was interested in assessing the impact of an appointment system on the length of time (i.e. cycle time) patients needs to spend at the polyclinic if only a selected proportion of patients were given appointments. They were also interested to assess if explicit allocation of specific doctors to see only patients with nonchronic conditions does help to lower overall patient cycle time.

As there were multiple patient appointment and patient-doctor allocation strategies that need to be evaluated in addition to uncertainty over their relative impact on the patient cycle times, discrete event simulation modelling was employed to evaluate these strategies and to provide evidence to support the management's decision-making process. Experimentation of these strategies through pilot scale implementation would be impractical as it could be operationally disruptive to the patients involved if the benefits of the strategies do not materialize. There is also concern over the ability of the existing manpower and equipment resources to cope with the demand of full-scale implementation of any of the abovementioned strategies.

\section{BACKGROUND AND RELATED WORK}

Scheduling of patient appointments entails assignment of specific time to patient at which the latter will receive care by the health service provider. Essentially, the ultimate goal of patient appointment scheduling is to allow patients to have timely and convenient access to necessary health services. A patient who is scheduled for an appointment faces two types of access delays. First, there is delay attributed to time interval between a patient's request of appointment time and the time of scheduled appointment. Second, there is also delay attributed to time interval between time of patient's scheduled appointment and the time the patient is actually served by the service provider. In the literature, both delays have been commonly known as indirect (virtual) and direct (captive) waiting time respectively. A welldesigned appointment system achieves small direct waiting times for unscheduled patients (especially those require urgent medical attention) without increasing the direct waiting times of scheduled patients or lowering manpower resource utilization. Moreover, an effective appointment scheduling system can also help to facilitate work flow, reduce crowding at waiting areas, and allow healthcare systems to accede to preferences of patients and service providers during the process of matching demand to supply.

In any outpatient clinics, appointment management is inherently complex due to two conflicting operational requirements. First, these clinics need to realize adequate manpower utilization level in accordance to management expectation. Second, they must also ensure that their patients have timely access to appropriate health services, which is critical for realizing good medical outcomes and patient satisfaction. In addition, several factors have to be taken into consideration in development of appointment schedules of patients. These factors include patient arrival, service time variability, preferences of patients and health service providers. 
Clearly, an effective schedule of patient appointments that can achieve a good balance among the aforementioned operational requirements with account of the inter-relationships among the entities (i.e. doctors, patients, nurses, others) is not intuitive.

In the early 1950s, it was [3, 4] concluded from field research that appointment scheduling problem can be studied most effectively using simulation. Under the assumption of same service time distribution for all patients, the author proposed the "individual appointment scheduling rules” in which the first $k$ patients are scheduled to arrive at the start of the day with subsequent patients scheduled at intervals equal to their expected service time. Based on a simulation study, the author concluded that a scheduling rule with $k=2$ often performs well in most instances. The results of his work were subsequently verified [5] in a much larger study where the authors introduced other good scheduling rules when the characteristics of patients are similar in a given clinic session. In another case study [6], discrete service time distributions of patients were employed to formulate the appointment-scheduling problem as a discrete time Markov chain model which allowed system state probabilities to be computed numerically. The authors used these probabilities to calculate various performance measures in their evaluation of different appointment strategies. Another comparative study [7] also assessed the impact of ten different scheduling rules on waiting times of patients and idle times of doctors through simulation experiments. In another work [8], simulation experiments were also employed to evaluate two methods of outpatient appointment scheduling. Recently, a simulation study [9] was performed to determine optimal number of planned appointments in different operational requirements and consult room configurations at specialist outpatient clinics. Alluding to a similar problem which aimed to improve patient care, a Modular Coloured Petri Net with changeable structure (MCPN-CS) workflow modelling method [10] was developed to better clinical pathway in disease treatment with deadlock avoidance feature.

From the above discussion, it is apparent that most the existing work in the literature focused on development of appointment scheduling rules or methods. To the best of the authors' knowledge, there is limited study on the impact of different proportions of appointment patients on the cycle times of appointment and walk-in patients in an outpatient clinic environment. Similarly, there is also limited study on the impact of allocating a number of doctors to condition-specific patients on the cycle times of these and other patients. To fill this research gap, this paper aims to offer technical insights on the effectiveness of different appointment apportionment and patient-doctor allocation strategies on the patient cycle times.

\section{METHODS AND MODEL}

Similar to previous work in the literature, this project entails development of a discrete-event simulation model to represent the interaction of entities (patients, doctors, nurses, etc.) in the polyclinic of interest. Essentially, this simulation model serves as an evaluation platform in determination of good appointment apportionment and patient-doctor allocation strategies that keep patient cycle times to the minimal. Before the task of simulation model development is embarked, it is crucial to establish understanding of the journey of patients at the polyclinic via process mapping. As such, we describe in the following section the sequence of visits made by the patients to different stations within the polyclinic of interest during each episode of care from the time they enter polyclinic and to when they exit polyclinic.

\subsection{Process mapping of polyclinic patients}

Upon arrival at the polyclinic, a patient will proceed to one of the two self-service kiosks where he or she will select the required service and then be issued a queue ticket. The services 
available for selection at the kiosk include seeing of doctors, nurses, and laboratory test. At the polyclinic, majority of the patients are walk-in patients with the intention of seeing the doctors. The rest of the patients may require seeing the nurses for health services like vaccination, counselling, etc. Alternatively, patients may also require laboratory tests prior to their doctor consultations.

After collecting their queue tickets, the patients then proceed to their respective stations to wait for their turns. Majority of the walk-in patients who wish to see doctors will wait at the waiting area adjacent to the registration counter till their queue numbers are called. The registration process basically entails verification and electronic entry of patient particulars by the registration counter staff. The support staff at the registration counter will then perform the necessary logistic arrangement of retrieving the patients' medical records and delivering them to them to their respective doctor consultation rooms. Once the registration process is completed, each of these walk-in patients will then proceed to the waiting area outside the consultation room of the doctor whom the patient has been assigned to. After a patient has seen the doctor, the former may move on to the pharmacy where the patient will drop the prescription form and wait for his/her turn to collect the medication. In another scenario, the patient may need laboratory tests after seeing the doctor. When this happens, the patient will then proceed to the laboratory to undertake the necessary tests before returning to see the same doctor for review of test results. After this review, the patient may then proceed to the pharmacy to collect his or her prescription.

At the pharmacy, there are three key processes, which must take place before a patient receives his or her medication. First, there is the ordering process where pharmacy staff will enter electronically the prescription of a patient and subsequently print out the medication labels which contain patients' particulars, names of their drugs and their respective dosages. After the ordering process is completed, another pharmacy staff will undertake the filling process where medications will be picked from their respective shelves, packed and consolidated. Lastly, there is the dispensing process where the pharmacy staff will call the patient and dispense the consolidated medications to the patients with all the necessary instructions. Once a patient has collected the medication at the pharmacy, he or she will move on to make payment at the cashier counter. There are two options for patients who wish to make payment at the polyclinic. Patients may wait for their respective turns to be called up to make payment at the cashier counter. Alternatively, the patients may proceed to the express cashier counter to make the payment as soon as an available cashier is ready to serve these patients. See Fig. 1 for an overview of a walk-in patient's journey at polyclinic from the point of arrival to exit.

To this end, it is important to highlight that majority of the polyclinic patients are served on a first-in-first-out (FIFO) basis at the all stations that include the self-service kiosk, registration counter, laboratory, doctor consultation room, pharmacy and cashier. However, the polyclinic does give priority to a group of privileged patients who include those wheelchair bound patients and regular blood donors. This means that this group of priority patients will be served earlier at any of the stations within the polyclinic compared to other patients waiting in queues at the same stations. Since patients can opt to make payment at the express cashier counter, they can spend minimal waiting time for payment if they do not wish to wait in queue at the cashier counter. As such, the patient cycle time of interest is defined in the rest of this paper as the interval between the instant a queue ticket is issued to the patient at the self-service kiosk and the point when the patient leaves the pharmacy after collection of medication. For a patient with no prescriptions, the cycle is defined as the interval between the instant a queue ticket is issued to the patient at the self-service kiosk and the point when the patient completes the consultation process with the doctor. 


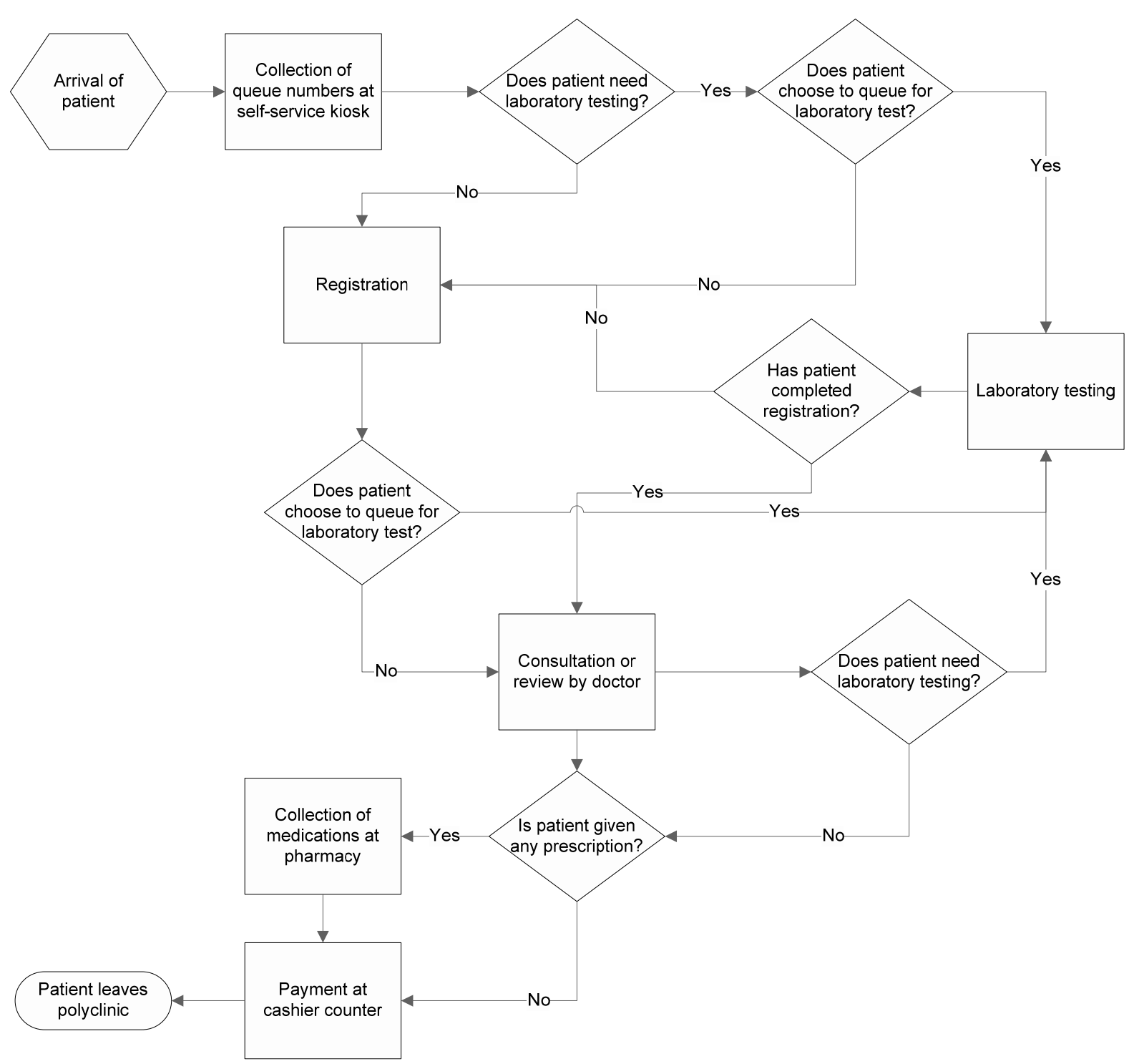

Figure 1: Journey of a patient at polyclinic.

\subsection{Simulation model development}

Regardless of the systems that are represented in discrete-event simulation, five key steps have to be performed before the resultant simulation models can be employed to evaluate the effectiveness of potential process improvement strategies. These five steps, their respective details, and how we employed them in our simulation model developmental effort are concisely described as follows.

\section{Definition of problem scope and objective}

This is to ascertain the boundary of the system to be represented by the simulation model and the goal to be accomplished from the process analysis. In this project, we accounted for the processes which took place between the arrival of patients at the self-service kiosks and their collection of medications at pharmacy in our problem analysis and model development work. For patients with no prescriptions, the processes that were accounted for included those which took place between arrival of patients at the self-service kiosks and completion of their respective consultation processes with the doctor. The project goal was to study the impact of different appointment apportionment and patient-doctor allocation strategies on patient cycle times. 
2 Identification of simulation model inputs

This involves identification of processes that make up the system of interest and the corresponding process data that are needed to represent the system realistically. To simulate the journey of patients who visit the polyclinic, key processes that needed to be accounted for in the simulation model included the arrival of patients, registration, activities that occur at laboratory testing, doctor consultation and pharmacy, and physical movement between stations in polyclinic. In addition, the profiles of the patients who visited polyclinic had to be accounted accordingly. These profiles include proportion of patient types (priority patients or normal patients), their medical conditions (chronic or others patients), proportion of patients who visit laboratory prior to the registration process, proportion of patients who need to go for laboratory tests after doctor consultation, etc.

3 Collection of data for model inputs

For required model inputs that are captured in electronic database, this step entails retrieval of necessary information from the database. If the required data is not available in the database, a time motion study (pioneered by industrial engineer F. W. Taylor) may have to be performed to collate the necessary information. In our work, the consolidated process parameters that were either based on electronically archived one-week operational data (i.e. historical data) or data collected via a time motion study performed over five day period were tabulated in Table 1. One assumption that we made in our simulation model development was that these process parameters such as service rate distributions of registration counter staff, laboratory technicians, doctors, etc were representative of underlying processes and time-independent. This assumption was endorsed by the polyclinic operations staff who felt the above data captured the variations of their processes and were adequately representative of the process characteristics. To this end, it was important to highlight the probability distributions of arrival patterns and services rates that were used to represent all processes of interest at the polyclinic were based on collated empirical data.

4 Development of simulation model

This entails computer coding that is typically done using programming languages or simulation software package. A simulation model that represented the flow of patients at polyclinic was developed using Arena (Professional Edition). Essentially, this software permits modular representation of processes where the sequential flow of patients to these processes can be predefined accordingly to simulate the historical-based patient flow in polyclinic.

\section{$5 \quad$ Validation of simulation model}

This is to ascertain that the resultant simulation model is representing the actual system of interest realistically. Based on the reported simulation model validation techniques in the literature [11], three key steps were performed to ascertain operational validity of resultant simulation model. First, we verified from the model output that the chronological sequences of process flow of all patients were in accordance to the characteristics of the patients. Second, we performed a subjective validation study [12] where we verified that the histograms of four key parameters (i.e. times spent by patients from kiosk to registration, kiosk to laboratory, registration to consultation and registration to pharmacy) generated by the simulation model matched closely to the corresponding histograms, which were based on polyclinic's recent one-month performance. Third, we also performed statistical tests to compare mean values of the above four key parameters generated by the simulation model with corresponding means values of polyclinic's one-month data. Based on the overall results of the abovementioned validation steps and the endorsement by clinic manager of the polyclinic, it was affirmed that the simulation model represented the process flow within the polyclinic of interest realistically. 
Table I: Input of polyclinic simulation model.

\begin{tabular}{|l|l|l|}
\hline Process & Parameter & Data Source \\
\hline $\begin{array}{l}\text { Arrival of patients at the } \\
\text { self-service kiosks }\end{array}$ & $\begin{array}{l}\text { Patient inter-arrival interval (length of time } \\
\text { between arrivals of consecutive patients) }\end{array}$ & Historical data \\
\hline Post-kiosk activity & $\begin{array}{l}\text { Proportion of patients who visit laboratory } \\
\text { prior to the registration process }\end{array}$ & Historical data \\
\hline Registration & $\begin{array}{l}\text { Registration time interval (length of time } \\
\text { between a patient's queue number is called and } \\
\text { the instant patient leaves the registration } \\
\text { counter) }\end{array}$ & Historical data \\
\hline Laboratory testing & $\begin{array}{l}\text { Time interval at the laboratory (length of time } \\
\text { between a patient enters and leaves a } \\
\text { laboratory) }\end{array}$ & Time motion study \\
\hline Consultation of doctor & $\begin{array}{l}\text { Time interval at the consultation room (length } \\
\text { of time between a patient enters and leaves a } \\
\text { consultation room) }\end{array}$ & Historical data \\
\hline Post-consultation activity & $\begin{array}{l}\text { Proportion of patients who need to go for } \\
\text { laboratory tests after doctor consultation }\end{array}$ & Historical data \\
\hline $\begin{array}{l}\text { Prescription ordering at } \\
\text { pharmacy }\end{array}$ & $\begin{array}{l}\text { Ordering time interval at the pharmacy (length } \\
\text { of time between a prescription is picked up by } \\
\text { an ordering staff and the instant when the latter } \\
\text { completes the ordering process) }\end{array}$ & Time motion study \\
\hline $\begin{array}{l}\text { Prescription filling at } \\
\text { pharmacy }\end{array}$ & $\begin{array}{l}\text { Filling time interval at the pharmacy (length of } \\
\text { time between the point when filling staff starts } \\
\text { collecting medications for a prescription and } \\
\text { the instant when the staff completes the filling } \\
\text { process) }\end{array}$ & Time motion study \\
\hline $\begin{array}{l}\text { Dispensing time interval at the pharmacy } \\
\text { consultation of doctor and } \\
\text { dispensing at pharmacy } \\
\text { patient und the instant when the staff completes } \\
\text { dispensing the medications to the patient } \\
\text { concerned) }\end{array}$ & $\begin{array}{l}\text { Proportions of patients who visit polyclinic due } \\
\text { to chronic, acute and other medical conditions } \\
\text { which have distinct laboratory testing, } \\
\text { consultation and dispensing time intervals. }\end{array}$ & Historical data \\
\hline
\end{tabular}

\section{RESULTS AND ANALYSIS}

With the validated model, we proceeded to evaluate the effectiveness of different appointment apportionment and patient-doctor allocation strategies. This evaluation exercise involved making the appropriate changes to the inputs of the simulation model to simulate an appointment apportionment or patient-doctor allocation strategy. The outputs of the amended simulation model were then used to project the impact of the strategy on the cycle times of patients. It was important to note that these outputs were based on 30 replications of simulation runs with each of these runs representing one full polyclinic operation day. Since the clinic manager felt that the one week of operational data was adequately representative of the polyclinic process characteristics, performance of 30 replication runs was adequate to generate outputs that were representative of the impact of any new strategy on patient cycle times. 


\subsection{Appointment apportionment study}

The polyclinic management was particularly interested in improving the clinical care of their chronic patients so that their medical conditions could be managed better. As such, they were keen to implement an appointment system for the chronic patients who constituted about $60 \%$ of the polyclinic daily attendance should a full-scale implementation of appointment system to all patients be present.

To emulate the arrival pattern of appointment patients in the simulation model, the latter was modified so that selected patients who were on appointment arrived at polyclinic 6 to 15 minutes before their scheduled appointments if they did not require pre-consultation laboratory testing. If the latter was necessary, the appointment patients were made in the simulation to arrive at the laboratory within the polyclinic 45 to 60 minutes before their respective scheduled appointment with the doctors. We made the assumption that patients placed on appointment would arrive early for their respective appointments in response to a penalty that would result in the appointment patients losing the priority to see the doctors once they were late. In short, appointment patients who were late would be required to queue with the walk-in patients to see their doctors.

To reflect the actual situation where appointment patients proceeded to the waiting area outside the doctor consultation rooms upon collection of queue numbers from the self-service kiosks, the simulation model was also modified to account for the revised journey of appointment patients. Similar to the assignment of walk-in patients to doctors at the polyclinic, the assignment of appointment patients was done with the goal of equalizing the load distribution among the doctors.

After the abovementioned amendments were made to the simulation model, three "whatif" analyses were performed where $100 \%, 50.8 \%$, and $25.4 \%$ of polyclinic's average daily patient attendance. These percentages were derived based on absolute numbers of appointment patients out of average daily attendance. It is also important to highlight that the $100 \%$ appointment system entailed both chronic and non-chronic patients being placed on appointment while the $50.8 \%$ and $25.4 \%$ of patients placed on appointment consisted of only chronic patients. For each of these three analyses, the simulation model was made to perform 20 replication runs and all the results were presented in Figs. 2 to 4 . Key performance metrics of interest include median and $95^{\text {th }}$ percentile cycle time of the overall patient population, appointment patients and non-appointment patients. Overall patient cycle time measures the cycle time of both appointment and non-appointment patients.

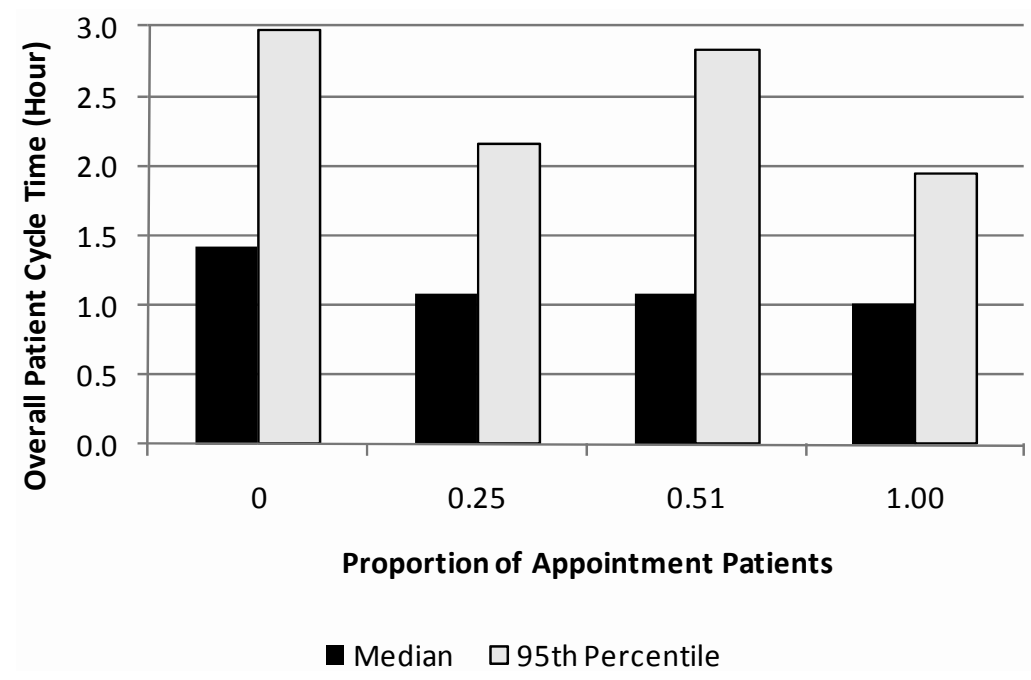

Figure 2: Impact of appointment patient proportions on overall patient cycle time. 


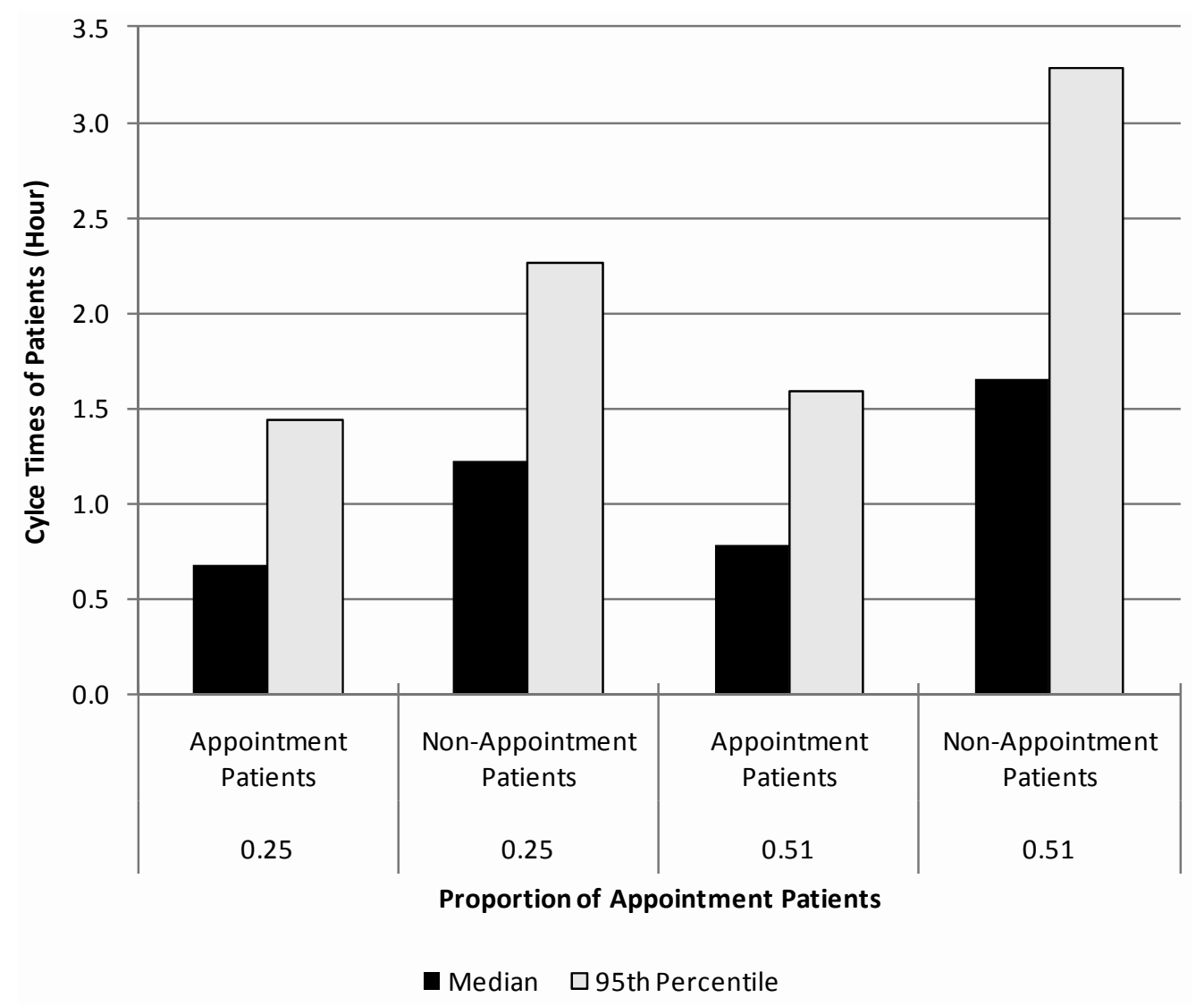

Figure 3: Impact of appointment patient proportions on cycle time of appointment and non-appointment patients.

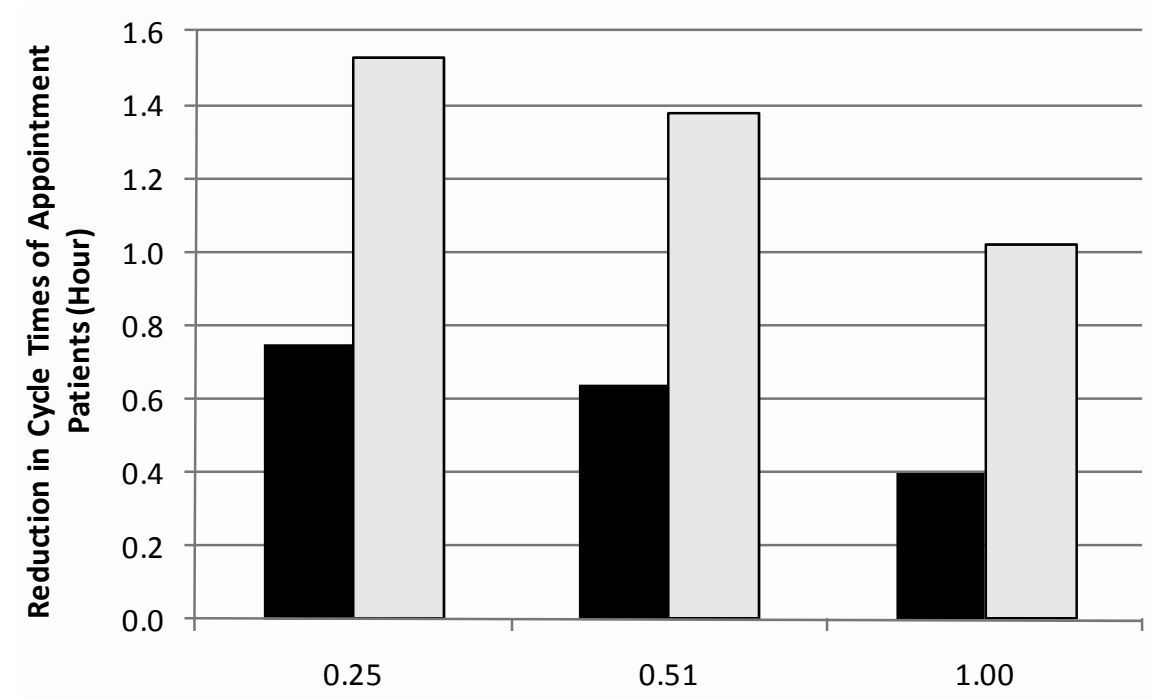

Proportion of Appointment Patients

- Median $\quad$ 955th Percentile

Figure 4: Impact of appointment patient proportions on the reduction in cycle time of appointment patients. 


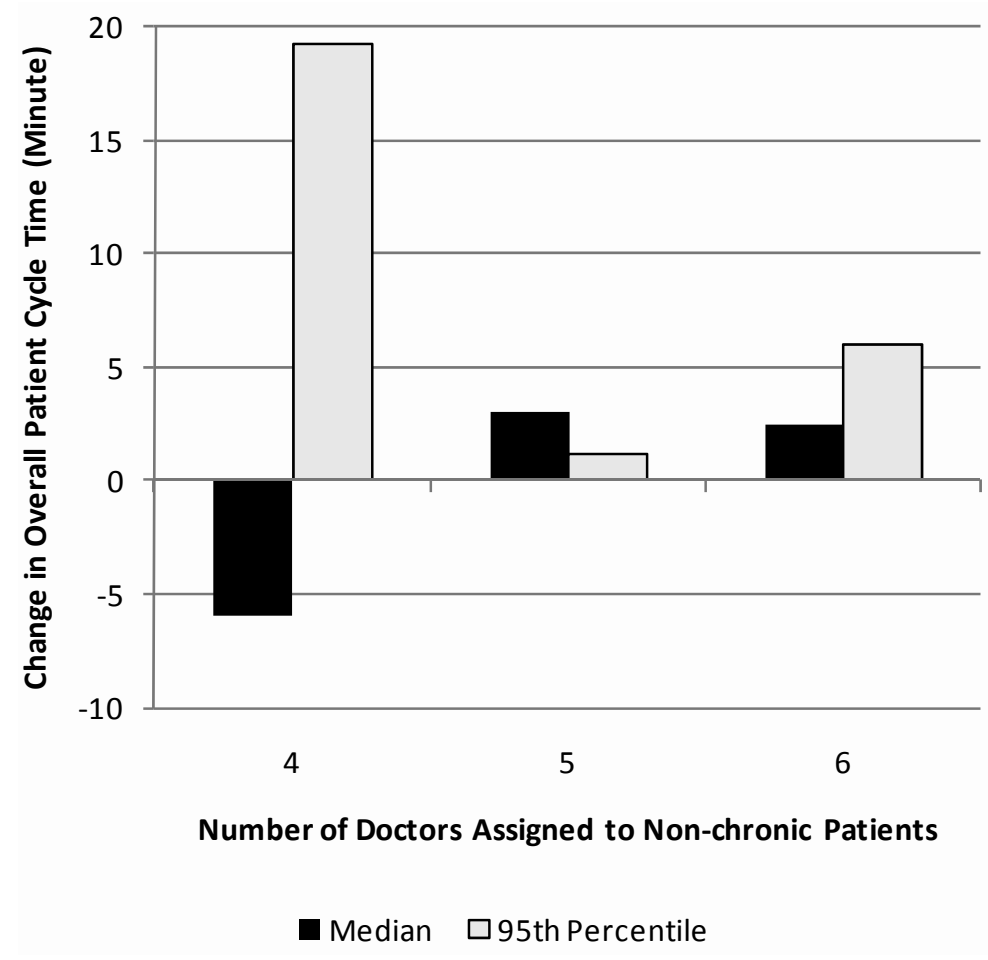

Figure 5: Impact of patient-doctor allocation strategies on overall patient cycle time.

In general, the benefit of lower median and $95^{\text {th }}$ percentile overall patient cycle time increased as the proportion of patients placed on appointment increased (see Fig. 2). Compared to the default condition where there were no appointment patients (i.e. proportion of appointment patients equals to zero), the maximum reductions (percentage change) in median and $95^{\text {th }}$ percentile overall patient cycle time were 0.40 hour $(28.2 \%)$ and 1.02 hour $(34.3 \%)$ respectively. Since maximum reductions in median and $95^{\text {th }}$ percentile overall patient cycle time were achieved when $100 \%$ of patients were given appointments, it is evident that existing manpower and equipment resources of the polyclinic were adequate to meet the operational needs of full appointment system without adverse consequences on the waiting times of patients. From the results, it was also apparent that appointment system offers an effective means of classifying patients so that those placed on appointments had shorter cycle times by up to 0.87 hour ( $52.7 \%$ reduction) and 1.69 hour (51.5 \% reduction) in terms of median and 95 percentile cycle times respectively at the polyclinic relative to the non-appointment patients (see Fig. 3). However, the magnitude of reduction in cycle times for appointment patients diminishes as the proportion of patients placed on appointment increases (see Fig. 4).

\subsection{Patient-Doctor allocation study}

Individual doctors in the polyclinic see a mixture of patients with either chronic or acute aliments on a daily basis. However, there was thought among the management team that there might be better patient management should there be separate dedicated teams of doctors attending to those with chronic and acute conditions respectively. The proportion of doctors allocated to the load of chronic and acute patients would be proportionate to the volume of patients in the respective categories. About $60 \%$ of the patients seek consultations at the polyclinic for chronic conditions. Since there are on average 12 doctors working at the polyclinic daily, the number of doctors who can be assigned to attend to non-chronic patients 
can be from four to six. However, there was uncertainty over the impact this strategy on the overall patient cycle time.

To assess the abovementioned impact, the simulation model was employed with one key amendment made to the original model setting. This entailed streamlining the flow of patients so that chronic patients were directed to a specific group of doctors while the rest of the doctors in the polyclinic attended to the non-chronic patients. We ran the model in three scenarios where four, five and six doctors were assigned to see only the non-chronic patients respectively and 20 replications were performed in each of these runs. The impact of these patient-doctor allocation strategies on the patient median and $95^{\text {th }}$ percentile cycle time was summarized in Fig. 5. Although the median patient cycle time was lowered by 6 minutes or $7 \%$ relative to that of existing system when four doctors were assigned to see only nonchronic patients, the corresponding $95^{\text {th }}$ percentile patient cycle time increased by 19.2 minutes or $10.8 \%$ relative to that of existing system. Increasing the doctor allocation to five and six for non-chronic patients also resulted in marginal increase in median and $95^{\text {th }}$ percentile overall patient cycle times. In view of this projection, it was concluded that assignment of patients to doctors based on their medical conditions does not improve overall patient cycle time.

\section{CONCLUSIONS}

Discrete event simulation modelling allows decision-makers the option to evaluate the effectiveness of potential process improvement strategies without committing excessive resources and avoiding possible disruptions to actual workflow. Our projections made through the DES model suggest that a partial patient appointment system offers an effective means of decreasing cycle times for those given appointments relative to the non-appointment patients. However, the magnitude of reduction in cycle times of appointment patients diminishes as the percentage of patients placed on appointment increases. It was also ascertained from analysis of model output that explicit assignment of patients to doctors based on their medical conditions was not optimal since it might unnecessarily prolong the cycle time patients. On the whole, these findings offered technical insights to the management of local polyclinic on the relative impact of different operating strategies that they had prior consideration for implementation. In addition, the findings had also given the polyclinic management confidence on the ability of their available resources to cope with partial or full appointment system.

Having a scientific method of evaluating the impact of changes in processes is useful in healthcare service delivery, particularly for those that entail costly interventions or measures and there is significant uncertainty over their effectiveness in achieving the desired outcomes. As health-care professionals work continuously to improve health-care operational efficiency in response to rising health-care costs and patient expectations, simulation models are excellent scientific frameworks that enable decision-makers to realize healthcare process enhancement.

\section{ACKNOWLEDGEMENTS}

This is a joint project between SingHealth Centre for Health Services Research and Bedok Polyclinic. The authors are grateful to Dr. Swah Teck-Sin, Ms. Jessie Neo and staff of Bedok Polyclinic for their discussions, comments and data exchanges during this work. 


\section{REFERENCES}

[1] Tan, H. L. (2006). Why those long polyclinic lines pose a health threat, Today, from http://www.pss.org.sg/main/content/view/86/2/, accessed on 01-09-2010

[2] Au-Yong, R.; Pang, A. (2009). Bedok Polyclinic tops with personal touch, The Straits Times, from http://www.asiaone.com/Health/News/Story/A1Story20090610-147438.html, accessed on 01-09-2010

[3] Bailey, N. (1952). A study of queues and appointment systems in hospital outpatient departments, with special reference to waiting times, Journal of the Royal Statistical Society, Vol. B14, 185-199

[4] Bailey, N. (1954). Queuing for medical care, Applied Statistics, Vol. 3, 137-145, doi:10.2307/2985372

[5] Ho, C. J.; Lau, H. S. (1992). Minimizing total cost in scheduling outpatient appointments, Management Science, Vol. 38, No. 12, 1750-1762, doi:10.1287/mnsc.38.12.1750

[6] Brahami, M.; Worthington, D. (1991). Queueing Models for Out-Patient Appointment Systems A Case Study, Journal of the Operational Research Society, Vol. 42, 733-746

[7] Klaseen, K.; Rohleder, T. (1996). Scheduling outpatient appointments in a dynamic environment, Journal of Operations Management, Vol. 16, No. 6, 384-396

[8] Vasilakis, C.; Kuramoto, L. (2005). Comparing two methods of scheduling outpatient clinic appointments using simulation experiments, Clinical and Investigative Medicine, Vol. 28, No. 6, 368-370

[9] Zhu, Z. C.; Heng, B. H.; Teow, K. L. (2009). Simulation Study of Optimal Appointment Number for Outpatient Clinics, International Journal of Simulation Modelling, Vol. 8, No. 3, 156-165, doi:10.2507/IJSIMM08(3)3.132

[10] Du, G.; Jiang, Z. B.; Diao, X. D.; Yao, Y. (2010). Reconfigurable Modelling with Deadlock Avoidance for the Clinical Pathway Based on MCPN-CS, International Journal of Simulation Modelling, Vol. 9, No. 2, 61-73, doi:10.2507/IJSIMM09(2)1.140

[11] Sargent, R. (1999). Validation and verification of simulation models, Proceedings of the Winter Simulation Conference, 39-48

[12] Sargent, R. (1996). Some subjective validation methods using graphical displays of data, Proceedings of the Winter Simulation Conference, 345-351 